\section{Thiopurine methyltransferase genotype and childhood acute lymphoblastic leukemia}

Mercaptopurine is an important component of treatment protocols for childhood acute lymphoblastic leukemia (ALL). The drug is inactivated by thiopurine methyltransferase (TPMT), a ubiquitously expressed, cytosolic enzyme that is subject to genetic polymorphism. Patients with intermediate or low TPMT activity-resulting from mutations in one or both copies of the TPMT gene-tend to achieve better event-free survival than those with normal TPMT activity. Stanulla et al. have recently investigated the effect of TPMT genotype in children receiving mercaptopurine during early treatment for ALL.

This ongoing study uses minimal residual disease analysis at two follow-up points, to facilitate risk-adapted treatment stratification in ALL patients aged 1-18 years. In the current analysis, 814 consecutive patients underwent TPMT genotyping and received a 4-week cycle of mercaptopurine.

Four patients were homozygous for a mutant TPMT allele and were excluded from the analysis. Of the remainder, 55 patients were heterozygous for allelic TPMT variants that resulted in lower activity of the enzyme. On day 78 , these children showed a significantly lower rate of minimal residual disease positivity than the 755 children with homozygous wild-type alleles (9.1\% vs $22.8 \%, P=0.02)$. This corresponded to an approximately 3 -fold reduction in risk for heterozygous individuals.

Concluding that TPMT genotype has a significant effect on response to mercaptopurine treatment, the authors emphasize the important role of minimal residual disease analysis in this setting.

Original article Stanulla M et al. (2005) Thiopurine methyltransferase (TPMT) genotype and early treatment response to mercaptopurine in childhood acute lymphoblastic leukemia. JAMA 293: 1485-1489

\section{Link between high human papillomavirus load and invasive cervical cancer}

Previous studies have suggested that a high human papillomavirus (HPV) load in cervical smears with normal cytology may be connected with the risk of developing dysplasia and carcinoma in situ. However, whether there is an association between high viral load in cervical smears and invasive cervical cancer is not yet known. Therefore, Moberg and colleagues from Sweden investigated smears from 62 cases diagnosed with invasive squamous cell carcinoma of the cervix, together with 501 control subjects.

The results showed that $45 \%$ of the cases and $6 \%$ of controls tested positive for HPV-16, with the odds ratio increasing with viral load, reaching a maximum of $51(\mathrm{P}=0.0001)$. HPV-31 was found in $8 \%$ of cases and $4 \%$ of controls, and HPV-18 plus HPV-45 was detected in $13 \%$ of cases and $6 \%$ of controls. The odds ratio for HPV-31 and HPV-18 plus HPV-45 was significant only for the highest viral load percentiles.

From these results, the authors conclude that an increasing viral load of HPV-16 in cervical smears increases the risk of developing invasive cervical cancer later on in life, and therefore viral load can be used as a risk marker for invasive cancer. However, further studies are needed to investigate the physical state and load of the virus, in order to determine the importance of these factors in the development of cervical cancer.

Original article Moberg M et al. (2005) High viral loads of human papillomavirus predict risk of invasive cervical carcinoma. Br J Cancer 92: 891-894

\section{Bortezomib and pegylated liposomal doxorubicin: phase I trial in patients with advanced hematologic malignancies}

A recent phase I trial has demonstrated that bortezomib, in combination with pegylated liposomal doxorubicin, can be safely administered to patients with advanced hematologic malignancies. The study also provides preliminary evidence of the antitumor activity of this proteasome inhibitor/anthracycline regimen.

Orlowski and co-workers administered escalating doses of bortezomib $(0.90,1.05,1.20$, $1.30,1.40$ and $1.50 \mathrm{mg} / \mathrm{m}^{2}$ ) to successive cohorts of 4-16 patients, all of whom had a confirmed hematologic malignancy. The drug was given intravenously on days 1, 4, 8 and 11 , followed by a 10-day rest period. Pegylated liposomal doxorubicin $\left(30 \mathrm{mg} / \mathrm{m}^{2}\right)$ was given as 